\title{
Emotional Regulation in Amyotrophic Lateral Sclerosis
}

\section{Bungener $\mathbf{C}^{1 *}$, Unglik $\mathbf{J}^{1,2}$ and Lacomblez $\mathrm{L}^{2,3}$}

${ }^{1}$ Laboratory of Psychopathology and Health Psychology - EA 4057, Sorbonne Paris Cité, Université Paris Descartes, Paris, France

${ }^{2}$ Neurology Department, AP-HP, Pitié-Salpêtrière Hospital, Paris, France

${ }^{3}$ CNRS, INSERM, Biomedical Imagery Laboratory, CIC-1422, Sorbonne Universités, UPMC Université Paris 6, Paris, France

Amyotrophic lateral sclerosis (ALS) is a progressive neurodegenerative disorder involving motor neurons of the cerebral cortex, brain stem and spinal cord. The loss of motor neurons quickly generates a progressive muscle paralysis usually leading to death from respiratory failure in 3-5 years. The cause of the disease is unknown and there is currently no effective treatment. Emotional regulation has not often been studied in ALS. Most published studies have assessed the psychopathological manifestations involved in this disease, essentially depression and less frequently anxiety [1]. They have shown that major depressive or anxious episodes are not frequent in ALS patients, although moderate depressive or anxious symptoms are often observed [2], but less frequently than in other neurological diseases like multiple sclerosis or Parkinson's disease [3]. Thus depressive symptoms are not correlated to the duration or the severity of the disease [4]. However anxiety symptoms appear to be present more often in the months following the diagnosis, and then they decrease. When considering the rapid evolution and the dire consequences of ALS, these observations are surprising. In order to better understand how the patients cope with their disease, it seems interesting to investigate their coping mechanisms on one hand, and the emotional regulation processes involved on the other. Coping has been described by Lazarus and Folkman [5] as "the constantly changing cognitive and behavioural efforts to manage the specific external or internal demands that are appraised as taxing or exceeding the resources of the person". Different coping strategies are described which can be grouped into two main domains: problemfocused coping, where the person tries to adapt by modifying the stressful situation and emotion-focused coping, where the person tries to modify his cognitive and emotional state. Literature shows that problem-focused coping is considered a more efficient way to reduce stress, anxiety and depressive symptoms.

In a previous study with a population of 169 ALS patients, we observed that $50 \%$ of them presented depressive symptoms and $60 \%$, anxious symptoms, although scores were low for anxiety and very low for depression. Patients experienced more negative subjective emotions than positive ones, and preferentially used emotional regulation as a coping strategy [6]. Emotional regulation and palliative coping strategies were positively correlated with negative emotions, depressive and anxiety symptoms and apathy. Distraction and cognitive avoidance strategies were negatively correlated with anxiety, depression and apathy, and positively with positive emotions. Such strategies can be considered as adaptive ones. These results confirm that emotion-focused coping is related to more psychological distress, which has been observed in various somatic and neurological disorders. Baker's model of emotional processing divides it into five domains: emotional experience, emotional expression, labelling, linkage and awareness [7]. This model refines our comprehension of the patient's adaptation, and has been carried out in multiple sclerosis [8] for example and is being used with ALS patients.

The relative psychological well-being of ALS patients could be partially explained by the attitude of their caregivers. In fact the ALS caregivers play an important role while the patient is becoming rapidly physically dependent [9]. Studies have observed that the caregivers are often more anxious and depressed than the patients, even if their emotional state does not seem related to the duration or severity of the disease [10]. Thus, it seems necessary to assess not only the caregivers' psychological states, but also the interaction between the patients and the caregivers. In fact if the caregiver is anxious or depressed, the patient will probably feel that he is more of a burden. The dyadic coping model of Bodenmann [11] details how couples manage relational stress. In fact, dyadic coping is supposed to be a major predictor of how couples are dealing with chronic illness [12]. According to Bodenmann, dyadic coping concerns the strains that affect one partner of the dyad, as well as the efforts of both partners to handle the stressful events. It includes the stress signals of one partner, the verbal or nonverbal coping responses of the other partner, and coping efforts of both. Different kinds of dyadic coping are described, grouped into two main categories. A positive type of dyadic coping which corresponds to a supportive approach, a delegated type of dyadic coping and a common type in which one partner takes over daily tasks, communicates empathy and conveys solidarity. Finally, there is a negative form of dyadic coping characterized by hostility, with one partner disparaging, distancing himself, mocking, using sarcasm, or minimizing his partner's stress. Dyadic coping can also be ambivalent, with one partner providing support, but unwillingly, or of a superficial nature. In ALS, Olsson et al. [13] have observed the positive effect on one partner of a psychological intervention on the other partner of the dyad. We are currently conducting research to assess these variables in ALS patients and their spouses, and this is the topic of the Unglik's PhD.

\section{References}

1. Carvalho TL, Almeida LM, Lorega CM, Barata MF, Ferreira ML, et al. (2016 Depression and anxiety in individuals with amyotrophic lateral sclerosis: A systematic review. Trends Psychiatry Psychother 38: 1-5.

2. Rabkin JG, Goetz R, Factor-Litvak P, Hupf J, McElhiney M, et al. (2015) Depression and wish to die in a multi-center cohort of ALS patients. Amyotroph Lateral Scler Frontotemporal Degener 16: 265-273.

3. Lulé D, Kurt A, Jürgens R, Kassubek I, Diekmann V, et al. (2005) Emotional responding in amyotrophic lateral sclerosis. J Neurol 252: 1517-1524.

4. Thakore NJ, Pioro EP (2016) Depression in ALS in a large self-reporting cohort. Neurology 86: 1-8.

5. Lazarus R, Folkman S (1984) Stress, appraisal and coping. Springer, New York

6. Unglik J, Bungener C, Delgadillo D, Salachas F, Pradat PF, et al. (2016) Emotional feeling, coping strategies and apathy in ALS. Ann Neurodegener Disord 1: 1019

7. Baker R, Thomas S, Thomas PW, Gower P, Santonastaso M, et al. (2010) The emotional processing scale: Scale refinement and abridgement (EPS-25). J Psychosom Res 68: 83-88.

*Corresponding author: Catherine Bungener, LPPS, Université Paris Descartes, 71 avenue Edouard Vaillant, 92100 Boulogne, France, E-mail: catherine.bungener@parisdescartes.fr

Received October 17, 2017; Accepted October 25, 2017; Published October 31, 2017

Citation: Bungener C, Unglik J, Lacomblez L (2017) Emotional Regulation in Amyotrophic Lateral Sclerosis. Int J Neurorehabilitation 4: 294. doi: 10.4172/23760281.1000294

Copyright: (ㄷ 2017 Bungener C, et al. This is an open-access article distributed under the terms of the Creative Commons Attribution License, which permits unrestricted use, distribution, and reproduction in any medium, provided the original author and source are credited. 
Citation: Bungener C, Unglik J, Lacomblez L (2017) Emotional Regulation in Amyotrophic Lateral Sclerosis. Int J Neurorehabilitation 4: 294. doi: 10.4172/2376-0281.1000294

8. Gay MC, Bungener C, Thomas S, Vrignaud, P, Thomas P, et al. (2017) Anxiety, emotional processing and depression in patients with multiple sclerosis. BMC Neuro 17: 43.

9. Jakobsson LB, Ozanne AG, Nordin K, Nygren I (2017) A prospective study of quality of life in amyotrophic lateral sclerosis patients. Acta Neurologica Scandinavica.

10. Chen D, Guo X, Zheng Z, Wei Q, Song W, et al. (2015) Depression and anxiety in ALS: Correlations between the distress of patients and caregivers. Muscle Nerve 51: 353-357.
11. Bodenmann G (1997) Dyadic coping - A systemic-transactional view of stress and coping among couples: Theory and empirical findings. Eur Rev App Psychol 47: 137-140.

12. Berg CA, Upchurch $R$ (2007) A developmental-contextual model of couples coping with chronic illness across the adult life span. Psychol Bull 133: 920 954

13. Olsson AG, Markhede I, Strang S, Persson LI (2010) Well-being in patients with amyotrophic lateral sclerosis and their next of kin over time. Acta Neurologica Scandinavia 121: 244-250 